\title{
Risk Analysis of Rice Losses Caused by Typhoon for Taiwan
}

\author{
Li-Hua Lai \\ National Kaohsiung First University of Science and Technology \\ E-Mail: lihua@ccms.nkfust.edu.tw \\ Pei-Hsuan Wu \\ National Kaohsiung First University of Science and Technology \\ Mei Ho Institute of Technology \\ E-Mail: u9227909@ccms.nkfust.edu.tw
}

\begin{abstract}
This paper applies extreme value theory to the Taiwanese rice loss caused by typhoons to demonstrate how these new statistical tools can be used to improve quantitative risk management. The application of the extreme value theory to statistics allowed us to test models with data from the upper tail of a distribution. Using the block-max and peak-over-threshold approaches to extreme value modeling, we matched generalized extreme value distribution (GEVD) and generalized Pareto distribution (GPD) to agricultural losses caused by natural disasters, with Taiwanese data from 1971 to 2005 . We then evaluated the appropriateness of the upper tail fitting to loss data by comparing with standard parametric modeling that is based on lognormal and gamma distributions. The extreme value theory outperforms classical parametric fits for the largest observed historical losses. Finally, we draw particular attention to issues related to agricultural disaster and calculate the 90th, 95th, and 99th percentile of VaR and expected shortfall. These results could help the authorities or agriculture risk managers to check the applicable loss-compensation regulations.
\end{abstract}

Keywords: Generalized Extreme Value Distribution, Generalized Pareto Distribution, T-Year Return Level, Value-at-Risk, Expected Shortfall

\section{INTRODUCTION}

Natural disasters worldwide have caused great damage to crop production (Adams et al., 1998; Rosenzweig et al., 2002; Cheng et al., 2005). Rice production is largely concentrated in Asia, where it is considered to be the major source of food 
(Taylor, 1996; Luo et al., 1998; Oerke and Dehne, 2004). According to Taiwan Yearbook $2006^{1}$, rice was ranked as Taiwan's most valuable crop in 2005. Over a 30-year period, however, Taiwan was hit by an average of 3.3 typhoons per year. The Council of Agriculture (COA) of Taiwan reports that among the natural disasters causing total rice loss for the past three decades, $60 \%$ were due to typhoons, which brought heavy rainfalls and strong winds, leading to severe damage to crops and great property losses (Wu and Kuo, 1999; Lin and Jeng, 2000; Chang, 2002; Singleton et al., 2005; Cheng et al., 2005). Hence, in this empirical study, we only analyze rice loss caused by typhoons. Related rice losses caused by typhoons in Japan (Yamagata et al., 1988; Yamamoto et al., 2000; Ji et al., 2002; Yamamoto and Iwaya, 2006) and the Philippines (Lansigan et al., 2000) have also been investigated. Luo et al. (1998) attempted to apply the concept of risk assessment to estimate the probability of certain levels of rice-yield loss for five Asian countries. Abbaspour et al. (1992) developed a computer system incorporating a crop growth model to estimate the 20 year expected losses for wheat in the Peace River region of British Columbia, Canada.

Extreme losses have a direct influence on the assessment of loss distribution of rice caused by natural disasters. Thus, assessment of heavy-tailed distribution plays a significant role in estimating rice yield and loss. Note that extreme rice loss tends to be ignored (Hansen, 2004; Larsson, 2005; Muralidharan and Pasalu, 2006). This implies that such an assumption seems to be incompatible. This paper aims to use the extreme value theory and the modeling strategy; the latter focuses on the block-max and peak-over-threshold approaches to extreme value modeling. Thus, we fit the generalized extreme value and generalized Pareto distribution to data on rice loss caused by typhoons in Taiwan over the period 1971-2005 (Lai and Wu, 2006). Such data were included in the Taiwan Agricultural Yearbook ${ }^{2}$, Production Cost and Income of Farm Products Statistics, and Taiwan Area Agricultural Products Wholesale Market Yearbook. The objectives of this study are to examine the distribution type of rice losses caused by typhoons under extreme condition, and to propose a threshold or threshold value in order to modify the assumption that normality imposes a lower threshold value; after the existence of heavy-tail distribution from using the original data, and after determining the method for estimating the parameters of best fit, the generalized Pareto distribution can be employed and compared with previously employed traditional distributions for the rice loss.

\footnotetext{
1 Taiwan Yearbook 2006 is published by Government Information Office, Taiwan.

2 Taiwan Agriculture Yearbook is published by the Council of Agriculture, Taiwan.
} 
This paper is organized as follows: Section 2 summarizes the basic theories concerning the estimation of the extreme observations, largely following Gilli and Kellezi (2006). Section 3 describes the data and estimates the parameters of the loss distributions using maximum likelihood and analyses theoretical model that can fit the data. Section 4 interprets the results. We draw conclusions in Section 5.

\section{THE BASIC THEORY}

Estimating an extreme loss severity distribution from historical data is an important activity in risk assessment. Extreme value theory (EVT) provides methods for quantifying such events and their consequences in a statistical way. Generally, there are two related ways of identifying extremes in real data. Extreme value (EV) and generalized Pareto (GP) models are introduced that are central for the statistical analysis of maxima or minima and of excess over a higher or lower threshold. In the following subsections, the fundamental theoretical results underlying the block maxima and the threshold method are summarized. Most of the material in this section follows Gilli and Kellezi (2006).

\section{The Generalized Extreme Value Distribution (GEVD)}

The generalized extreme value (GEV) family describes the distribution of the maximum of the sets of observations. Suppose $\{X n\}$ is a sequence of independent and identically distributed (i.i.d.) random variables and $\mathrm{Mn}$ is the $\max \{\mathrm{X} 1, \ldots, \mathrm{Xn}\}$. Then if there exist constants $\mathrm{cn}>0$ and $\mathrm{dn} \in \mathrm{R}$ (a real number), (Mn - dn)/ $\mathrm{cn}$ is a centered and normalized maximum. If (Mn - $\mathrm{dn}) / \mathrm{cn} \stackrel{d}{\longrightarrow} \mathrm{H}$ (that is, converges in distribution to $\mathrm{H}$ ), for some non-degenerate distribution function $\mathrm{H}$, then $\mathrm{H}$ belongs to one of the three families of extreme value distribution functions (Lai and $\mathrm{Wu}, 2007$ ):

$$
\begin{array}{ll}
\text { Gumbel: } & \Lambda(x)=\exp \left(-e^{-x}\right), \text { for all } x \\
\text { Fréchet: } & \Phi_{\alpha}(x)=\left\{\begin{array}{cc}
\exp \left(-x^{-\alpha}\right), & x>0 \\
0, & x \leq 0
\end{array}, \alpha>0\right. \\
\text { Weibull: } & \Psi_{\alpha}(x)=\left\{\begin{array}{cc}
\exp \left(-(-x)^{-\alpha}\right), & x \leq 0 \\
1, & x>0
\end{array}, \alpha<0\right.
\end{array}
$$

where $\alpha$ is shape parameter. 
By taking $\xi=1 / \alpha$, Jenkinson (1955) and von Mises (1936) suggested the following one-parameter representation

$$
H_{\xi}(x)=\left\{\begin{array}{cc}
\exp \left(-(1+\xi x)^{-1 / \xi}\right), & \text { for } \xi \neq 0,1+\xi x>0 \\
\exp \left(-e^{-x}\right), & \text { for } \xi=0
\end{array}\right.
$$

of these three standard distributions, with $x$ such that $1+\xi x>0$. This generalization, known as the generalized extreme value (GEV) distribution, is obtained by setting $\xi=\alpha^{-1}$ for Fréchet distribution, $\xi=-\alpha^{-1}$ for the Weibull distribution and by interpreting the Gumbel distribution as limit case for $\xi=0$, and $\xi$ is shape parameter. If we introduce location and scale parameters $\mu$ and $\sigma>0$ respectively, we can extend the family of distributions. We define the GEV $H_{\xi, \mu, \sigma}(x)$ to be $H_{\xi}((x-\mu) / \sigma)$ and we say that $H_{\xi, \mu, \sigma}$ is of the type $H_{\xi}$.

\section{The Generalized Pareto Distribution (GPD)}

Another distribution that plays an important role in modeling extremely events is the generalized Pareto distribution (GPD). If we consider an unknown distribution function $\mathrm{F}$ of a random variable $\mathrm{X}$, we are interested in estimating the distribution function $\mathrm{Fu}$ of variable of $\mathrm{x}$ above a certain threshold $\mathrm{u}$. The distribution function $\mathrm{Fu}$ is so called the conditional excess distribution function and is defined as

$$
F_{u}(y)=P(X-u \leq y \mid X>u), \quad 0 \leq y \leq x_{F}-u
$$

where $\mathrm{X}$ is random variable, $\mathrm{u}$ is a given threshold, $\mathrm{y}=\mathrm{x}-\mathrm{u}$ are the excesses and $\mathrm{xF}$ is the right endpoint of $\mathrm{F}$. We verify that Fu can be written in

$$
F_{u}(y)=\frac{F(u+y)-F(u)}{1-F(u)}=\frac{F(x)-F(u)}{1-F(u)}
$$

Pickands (1975), Balkema and de Haan (1974) posed that for a large class of underlying distribution function $\mathrm{F}$ the conditional excess distribution function $F_{u}(y)$, for $u$ large, is well approximated by 


$$
F_{u}(y) \approx G_{\xi, \sigma}(y), \quad u \rightarrow \infty
$$

where

$$
G_{\xi, \sigma}(y)=\left\{\begin{array}{cc}
1-\left(1+\frac{\xi}{\sigma} y\right)^{-1 / \xi} & \text { if } \xi \neq 0 \\
1-e^{-y / \sigma} & \text { if } \xi=0
\end{array}\right.
$$

for $y \in\left[0,\left(x_{F}-u\right)\right]$ if $\xi \geq 0$ and $y \in\left[0,-\frac{\sigma}{\xi}\right]$ if $\xi<0 . G_{\xi, \sigma}$ is the so-called generalized Pareto distribution. By putting $\mathrm{x}=\mathrm{u}+\mathrm{y}$, the GPD can also be expressed as a function of $x$, i.e. $G_{\xi, \sigma}(x)=1-(1+\xi(x-u) / \sigma)^{-1 / \xi}$.

\section{DATA AND ESTIMATION}

\section{Data Descriptions}

The data used in this study are observations on 117 rice losses caused by typhoons in Taiwan in the years 1971-2005. By adjusting for inflation, the rice loss data were made comparable through the years; all monetary magnitudes reported in this paper are in 2001 New Taiwan Dollar (NT\$). The overall summary statistics for rice losses are shown in Table 1. From 1971 to 2005, the average loss amount caused by each typhoon was nearly NT\$20 million. The data are considerably skewed to the right; the skewness coefficient is equal to 5.4. The box-plot of losses is shown in Figure 1 (Lai and $\mathrm{Wu}, 2007$ ). The upper whisker and several outliers indicate the long-tailed behavior of the underlying data. The Jarque-Bera test applied to rice loss data leads to a clear rejection of null hypothesis of normality with a p-value of zero. Therefore, we are interested in these severe losses and try to capture the behavior of the loss tail by using the extreme value theory.

Table 1 Rice Losses Summary Statistics NT $\$ 1,000$

\begin{tabular}{ccccccc}
\hline Min & Max & Mean & Median & Standard Deviation & Skewness & Kurtosis \\
\hline 21 & $3,863,707$ & 197,732 & 24,849 & 482,417 & 5.4 & 35.3 \\
\hline
\end{tabular}

Source: Taiwan Agricultural Yearbook 


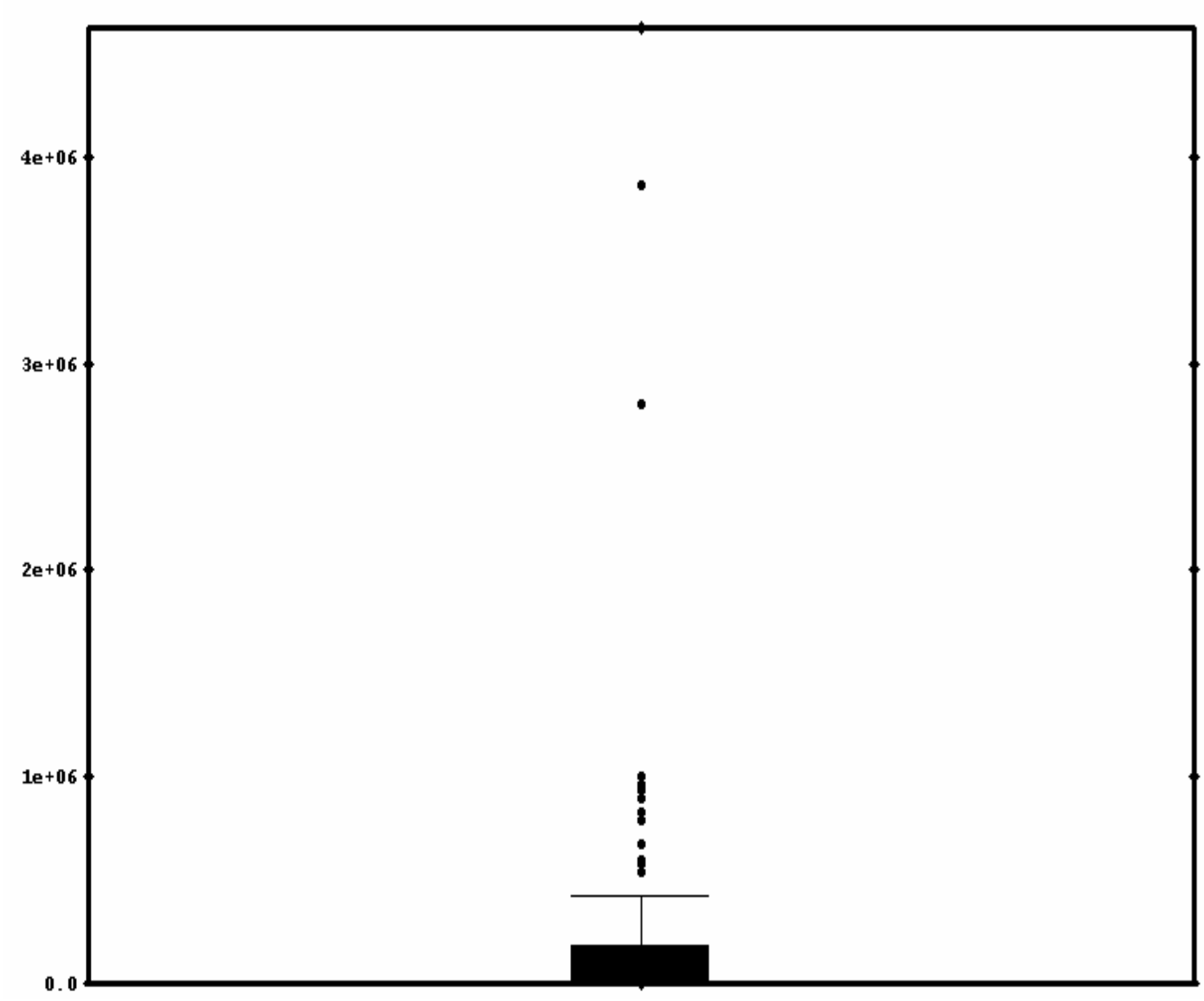

Figure 1 Box-Plot of losses

\section{GEVD Model of Rice Loss}

The cdf $F(x)$ corresponding to the GEVD is defined as

$$
F(x)=\exp \left(-\left(1+\xi\left(\frac{x-\mu}{\sigma}\right)^{-1 / \xi}\right) \quad \xi \neq 0\right.
$$

where $X$ (annual maximum loss) is random variable. $\mu, \sigma$, and $\xi$ are the location, scale, and shape parameters, respectively.

This analysis is based on the series of maximum annual typhoon damage on rice recorded in Taiwan, over the period 1971-2005; the data summary is shown in Table 2. The maximum likelihood estimates are summarized in Table 3. Since there were no typhoon damages on rice in 1983 and 1993, we dropped the data for these years. Hence, the source data is a set of 33 records of maximum annual rice loss caused by typhoons. 
Table 2 Data Summary Statistics for GEVD Model NT\$1,000

\begin{tabular}{ccccccc}
\hline Min & Max & Mean & Median & $\begin{array}{c}\text { Standard } \\
\text { Deviation }\end{array}$ & Skewness & Kurtosis \\
\hline 11,359 & $3,863,707$ & 544,636 & 311,254 & 789,978 & 3.2 & 11.1 \\
\hline
\end{tabular}

Source: Taiwan Agricultural Yearbook

Table 3 MLE for GEVD Model Fitted

\begin{tabular}{ccccc}
\hline location $\hat{\mu}$ & scale $\hat{\sigma}$ & shape $\hat{\xi}$ & K-S statistic & p-value \\
\hline $1.85 \times 10^{5}$ & $2.09 \times 10^{5}$ & 0.6837 & & \\
$\left(4.53 \times 10^{4}\right)^{*}$ & $\left(4.77 \times 10^{4}\right)^{*}$ & $\left(0.25 \times 10^{4}\right)^{*}$ & 0.07086 & 0.992 \\
\hline
\end{tabular}

* denotes s.e. of MLE

\section{GPD Model of Rice Loss}

In this section, we focuses on the statistical behavior of exceeds over a higher threshold. The practical problem is how to determine a "high enough threshold" or, likewise, the number $\mathrm{k}$ of upper extremes. To determine the optimal threshold value, we present the GPD estimate plot graphical tool. The choice of the number $\mathrm{k}$ of upper extremes can be supported visually by a diagram. Thereby, estimates are plotted against the number $\mathrm{k}$ of upper ordered values. If $\mathrm{k}$ is appropriate for the data, the values of the estimates stabilize around the true extremes and a plateau becomes visible. Figure 2 is a plot of the GP estimate versus k. It shows a plateau with right endpoint around $\mathrm{k}$ equal to 55. This evidence suggests processing the estimation on this number of upper extremes. Hence, by now, our analysis is based on 55 largest rice loss amounts over NT\$30,502 caused by typhoons in Taiwan, over the period 1971-2005. The data summary is shown in Table 4. In practice, we often use the following formula:

$$
G_{\xi, \sigma}(y)=1-(1+\xi y / \sigma)^{-1 / \xi}
$$

where Y (loss excess) is a random variable, $\sigma$ and $\xi$ are the scale and shape parameters, respectively. With excess number $\mathrm{k}$ set to 55 , the maximum likelihood estimates are summarized in Table 5 (Lai and $\mathrm{Wu}, 2008$ ). The results show that rice loss data are appropriate when using GPD model fitting, if enough data are available above a high enough threshold. 


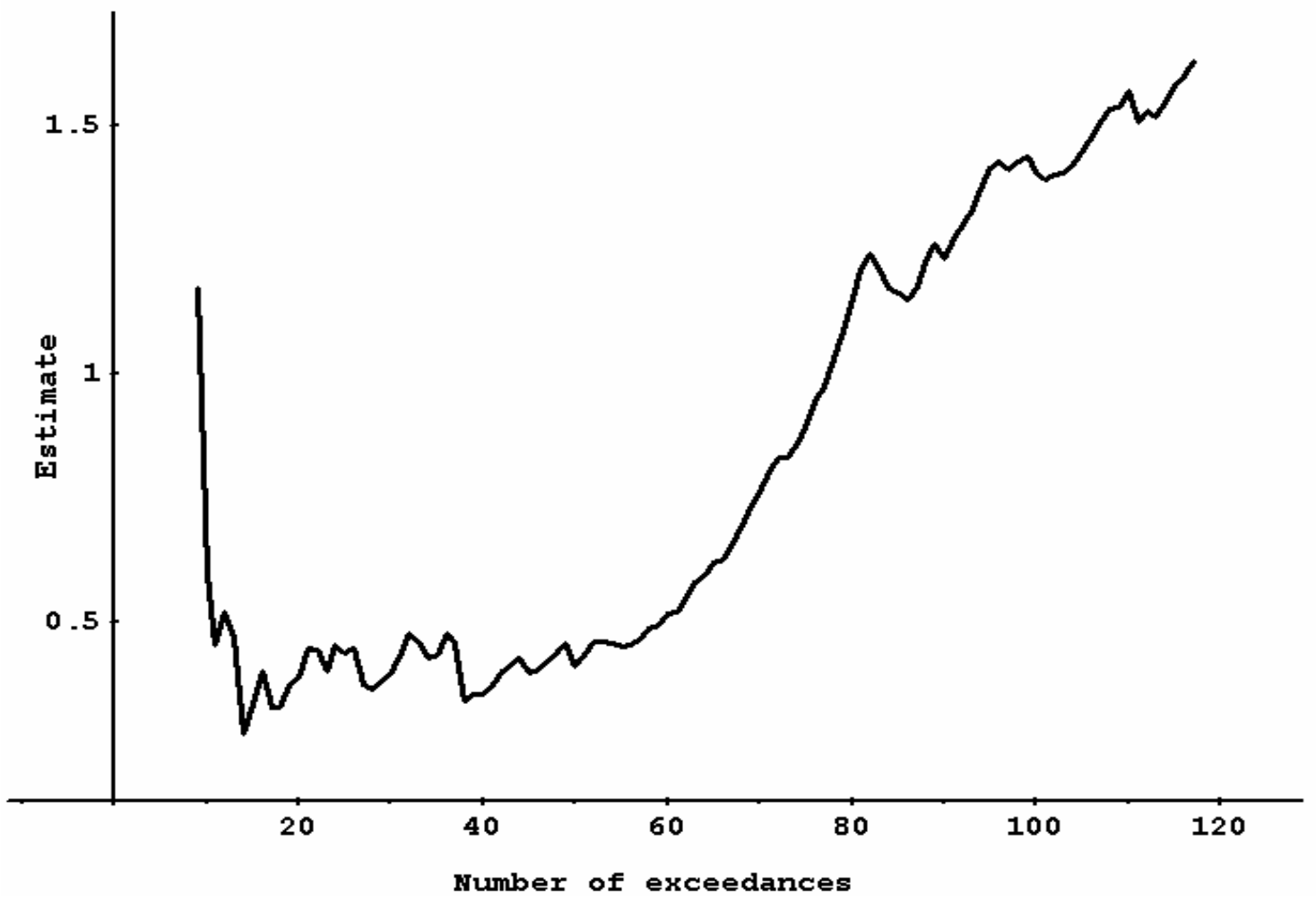

Figure 2 MLE estimates plotted against $k$

Table 4 Data Summary Statistics for GPD Model N NT1,000

\begin{tabular}{ccccccc} 
Min & Max & Mean & Median & $\begin{array}{c}\text { Standard } \\
\text { Deviation }\end{array}$ & Skewness & Kurtosis \\
\hline 33,813 & $3,863,707$ & $409,942.93$ & 196,272 & 642,883 & 3.97 & 18.17 \\
\hline Source: Taiwan Agricultural Yearbook & & &
\end{tabular}

Source: Taiwan Agricultural Yearbook

Table 5 MLE for GPD Model Fitted

\begin{tabular}{cccccc}
\hline$u$ & $k$ & $\hat{\sigma}$ & $\hat{\xi}$ & K-S statistic & p-value \\
\hline \multirow{2}{*}{30,502} & 55 & $2.15 \times 10^{5}$ & 0.4526 & & \\
& & $\left(4.98 \times 10^{4}\right)^{*}$ & $\left(0.1988 \times 10^{4}\right)^{*}$ & 0.04748 & 0.999 \\
\hline
\end{tabular}

denotes s.e. of MLE

\section{Models Checking}

We can visually assess how good the fit is by comparing probability density graphs with the histogram of sample data on a single chart and examining the probability-probability (P-P) plot of each fitted model separately. The P-P plot is a graph of the empirical cumulative distribution function (CDF) values plotted against 
the theoretical CDF values. It is used to determine how well a specific distribution fits to the observed data. This plot will be approximately linear if the specified theoretical distribution is the correct model. For GEVD model fitting, Figure 3 illustrates the probability density function of the fitted model that follows the shape of the data, especially at the tail. Moreover, the absence of a marked deviation of the P-P plot from the main diagonal in the unit square indicates that the fitted model is a good choice. We therefore conducted the testing of the generalized extreme models. The Kolmogorov-Smirnov test has been performed to check the compliance of the data with GEVD. The corresponding p-value shown in Table 3 suggests that GEVD is appropriate. Similarly, Figure 4 shows the same results of goodness-of-fit of the GPD model. Therefore, we are confident of the results as shown in Table 5.

\section{Comparison with Different Distributions}

Using block-max and excess data, we further tried to check the improvements achieved by using the GEVD and GPD instead of classical loss models, such as lognormal and gamma distributions. We provide a probability difference graph, Figure 5 , which is a plot of the difference between the empirical CDF and the theoretical CDF. This graph can be used to determine how well the theoretical distribution fits to the observed data and compare the goodness of fit of several fitted distributions. We can see that the GEVD and GPD fit the observed data better than the lognormal and gamma distributions. We are confident that our model selection offers the best approximation of the loss data; therefore, we can apply these probabilistic models to obtain the needed inferences.

\section{INTERPRETING THE RESULTS}

\section{Using GEVD Model to Measuring Extreme Risks}

For a wide range of application fields, the return level is a fundamental quantity to describe the behavior of the upper tail of a distribution. In extreme value analysis, it is often desirable to compute the size and the probability of T-year events, such as a hundred-year flood event. The T-year level $\mathrm{u}(\mathrm{T})$ is a threshold such that the mean number of excess over $\mathrm{u}(\mathrm{T})$ within the time span of length $\mathrm{T}$ is equal to 1 (Reiss and Thomas, 1997). After developing the GEVD model for rice loss, which is the annual maximum, we have applied this distribution to predict $\mathrm{u}(\mathrm{T})$. By using the following formula, T-year return level and excess times can be computed. 


$$
u(T)=F^{-1}(1=1 / T)
$$

which is the (1-1/T)-quantile of $\mathrm{F}$. Then we have

$$
P\left(X_{1}>u(T)\right)=1-F(u(T))=\frac{1}{T}
$$

where the T-year level $u(T)$ is exceeded by the observation in the given year with probability 1/T (Reiss and Thomas, 1997). Table 6 shows the 10-year, 20-year, and 50-year return level. These results could help the authorities or agriculture risk managers to build warning systems against typhoons that lead to rice damage and to check applicable compensation regulations.

Table 6 T-year Return Level Based on GEVD Model $\quad$ NT\$1,000

\begin{tabular}{ccc}
\hline 10-year return level & 20-year return level & 50-year return level \\
\hline $1.30347 \times 10^{6}$ & $2.20884 \times 10^{6}$ & $4.28395 \times 10^{6}$ \\
\hline
\end{tabular}

\section{Using GPD Model to Measuring Extreme Risks}

Next, we have applied this distribution to predict possible mean loss for each coming disaster. In this section, we will concentrate on two useful risk measures, which attempt to describe the tail of a loss distribution - VaR and expected shortfall. Value-at-Risk (VaR) is the qth quantile of the distribution F (Jorion, 2007; Lai and $\mathrm{Wu}, 2007)$

$$
\operatorname{VaR}_{q}=F^{-1}(q)
$$

where $F^{-1}$ is the inverse of $F$, and expected shortfall is the expected loss size, given that $\mathrm{VaR}$ is exceeded

$$
E S_{q}=E\left[X \mid X>V a R_{q}\right]
$$

Our goal in risk management is to estimate $\hat{\operatorname{VaR}} R_{q}$ and $\hat{E S_{q}}$ of rice loss measures. For a given probability $q>F(u)$, the VaR estimate is calculated in terms of GPD estimators, sample size $\mathrm{n}$, and excess numbers $N u$. The estimating formula is

$$
\hat{\operatorname{VaR}} q=u+\frac{\hat{\sigma}}{\hat{\xi}}\left(\left(\frac{n}{N_{u}}(1-q)\right)^{-\hat{\xi}}-1\right)
$$




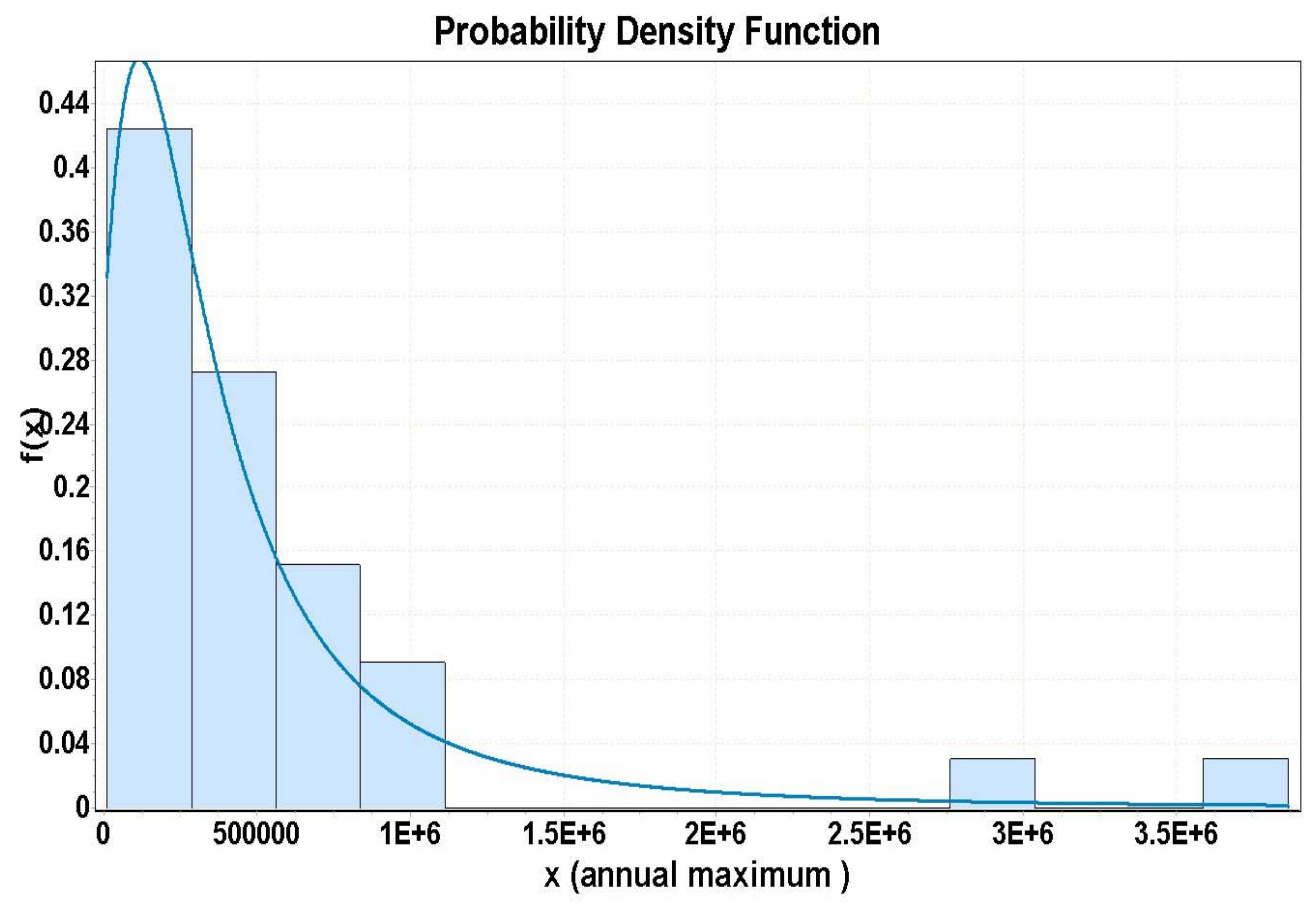

Histogram —Gen. Extreme Value

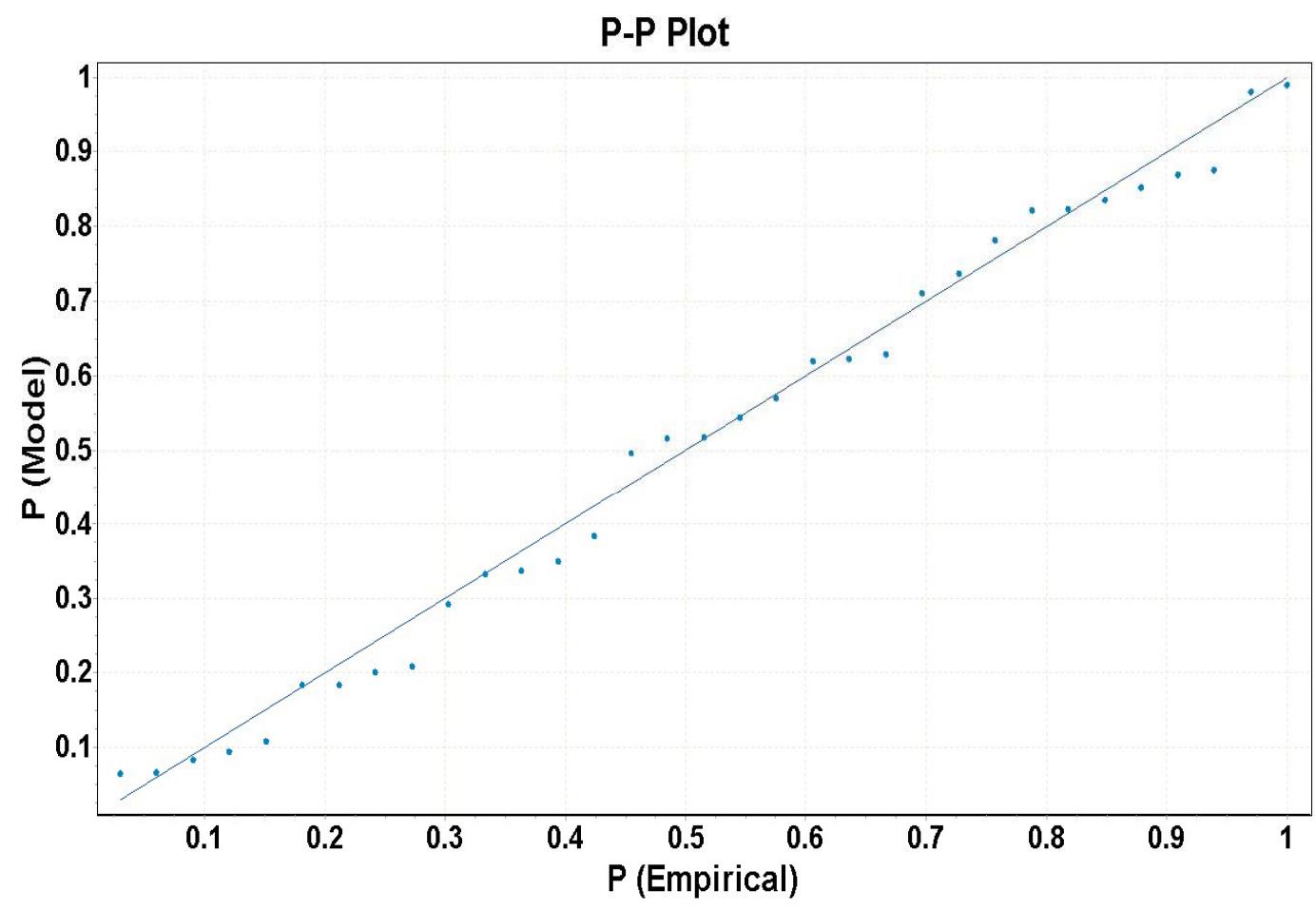

- Gen. Extreme Value

Figure 3 Probability density graphs with the histogram of sample data and P-P plot of fitted model of GEVD model 


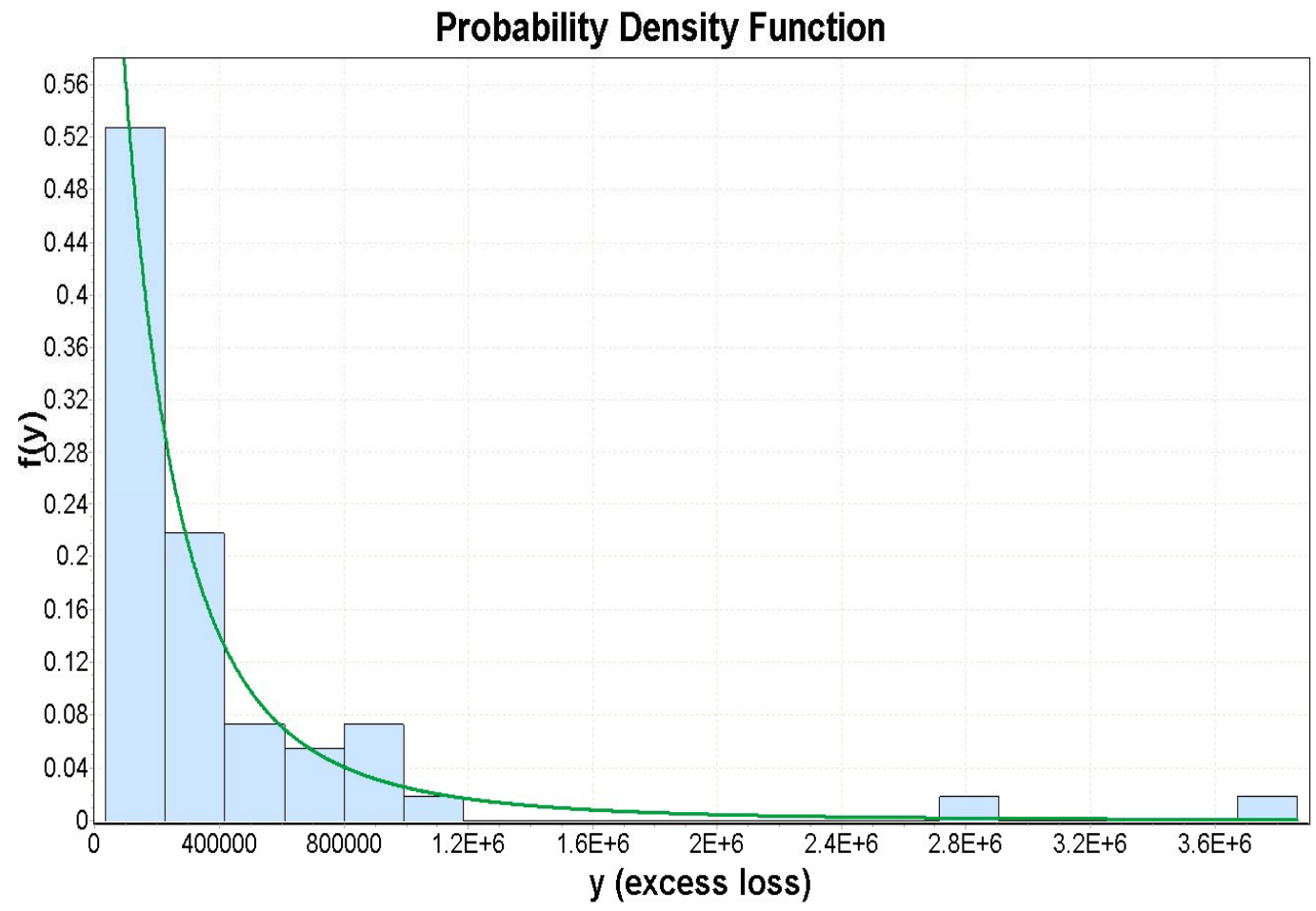

Histogram -Gen. Pareto

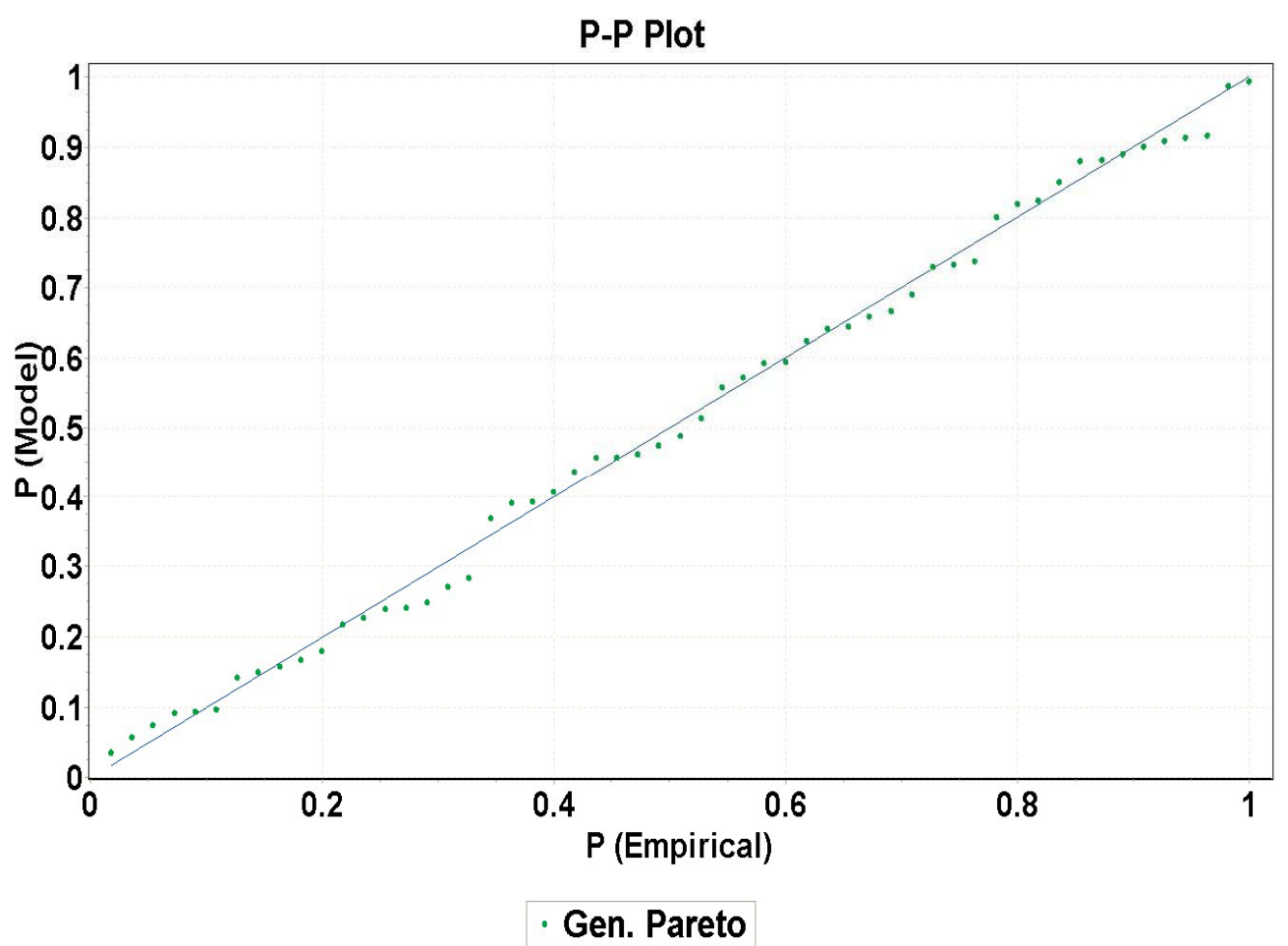

Figure 4 Probability density graphs with the histogram of sample data and P-P plot of fitted model of GPD model 


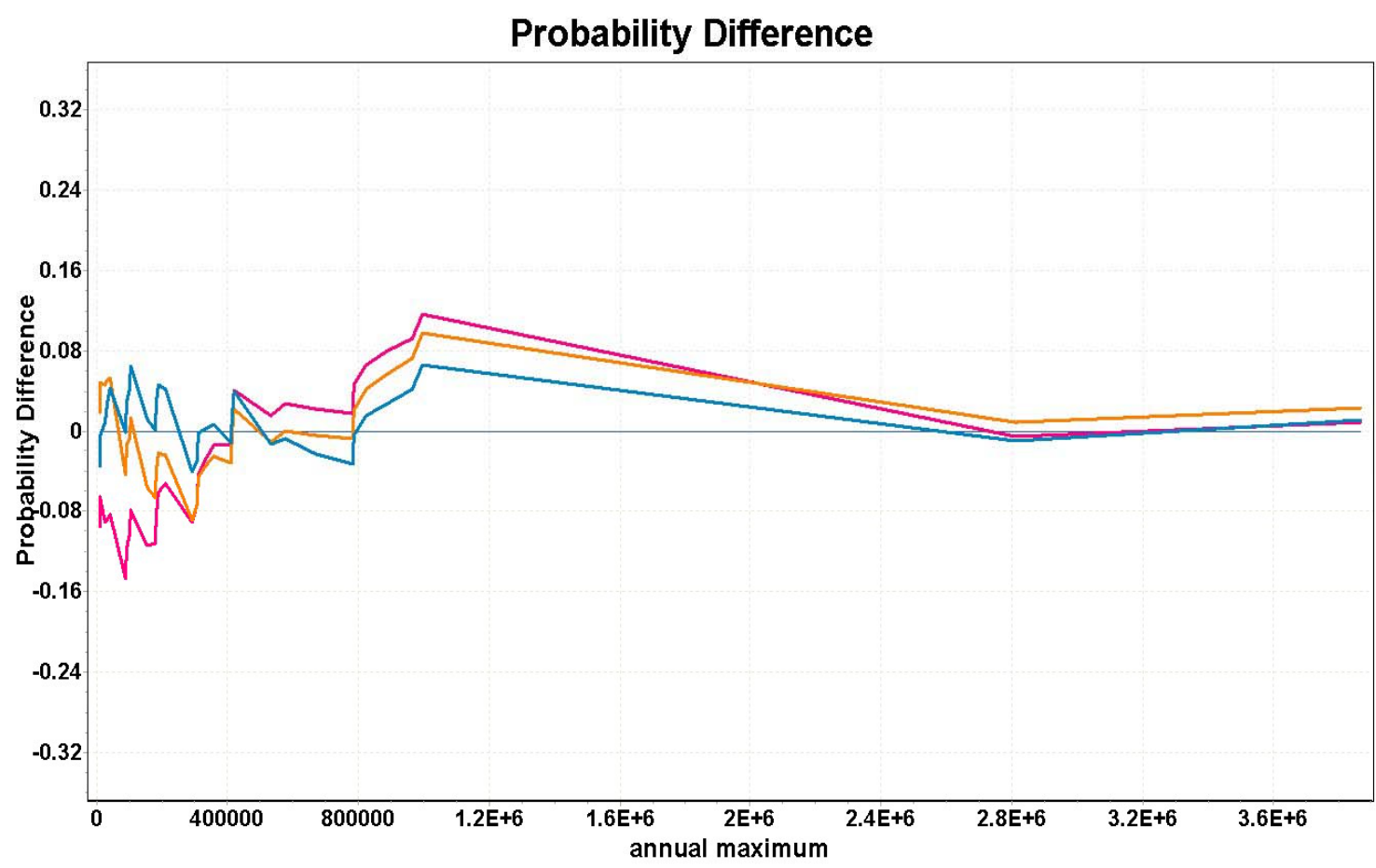

-Gamma - Lognormal - Gen. Extreme Value

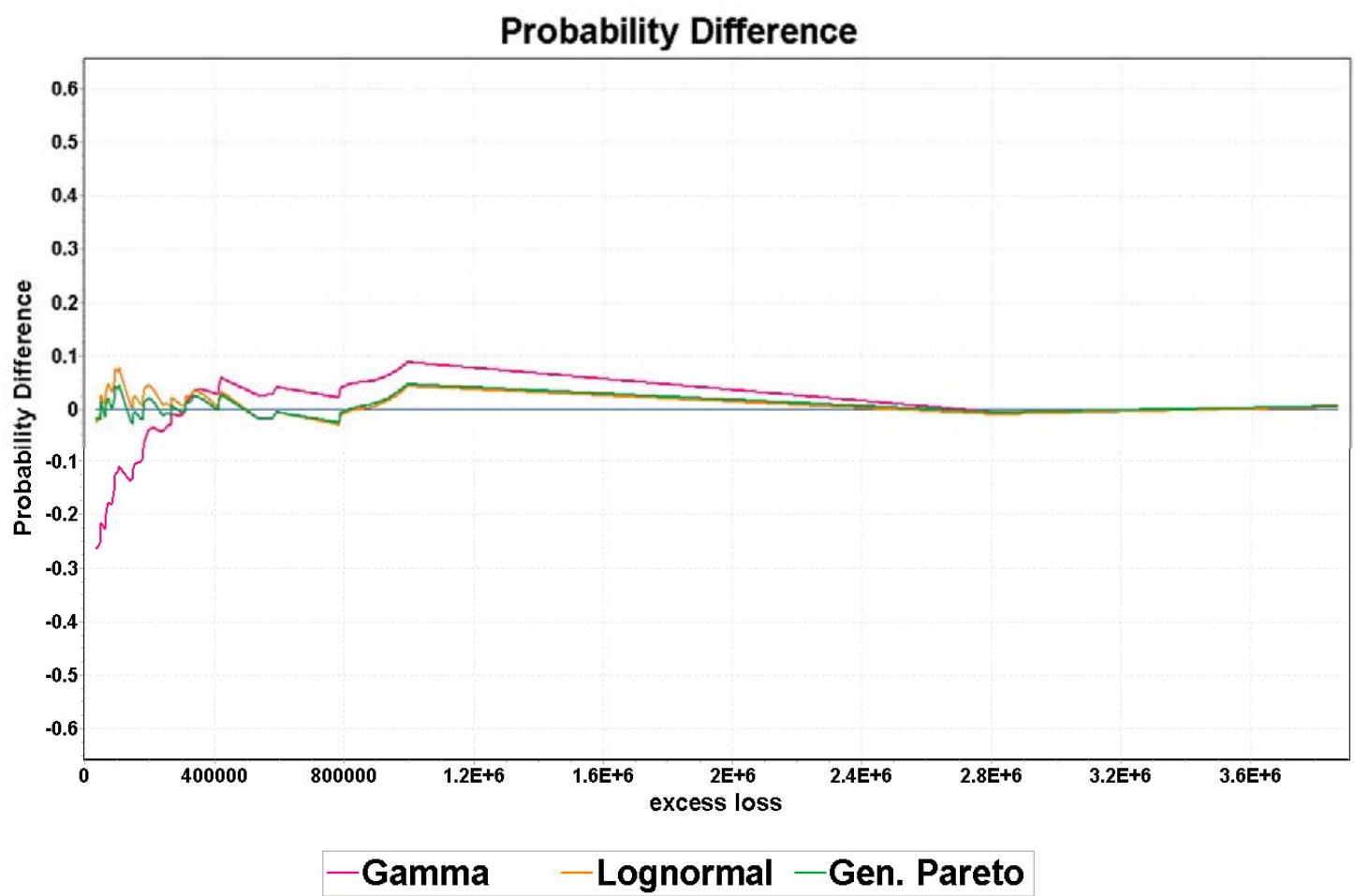

Figure 5 Probability different for various distributions fits 
Furthermore, we could get expected shortfall estimate using the following formula:

$$
\hat{E S}_{q}=\frac{\hat{V a R_{q}}}{1-\hat{\xi}}+\frac{\hat{\sigma}-\hat{\xi} u}{1-\hat{\xi}}
$$

In agriculture risk management, we are concerned with each natural disaster determination of the VaR for the damage and expected loss amounts over a cash relief threshold. Therefore, the $90^{\text {th }}, 95^{\text {th }}$, and $99^{\text {th }}$ percentile of VaR and ES are calculated, as shown in Table 7 (Lai and $\mathrm{Wu}, 2007$ ). These results could help the authorities and agriculture risk managers to check the applicable loss-compensation regulations.

Table 7 Point Estimates of VaRq and ESq for GPD Model

NT\$1,000

\begin{tabular}{cccccc}
\hline $\mathrm{VaR}_{0.90}$ & $\mathrm{ES}_{0.90}$ & $\mathrm{VaR}_{0.95}$ & $\mathrm{ES}_{0.95}$ & $\mathrm{VaR}_{0.99}$ & $\mathrm{ES}_{0.99}$ \\
\hline 377,383 & 941,144 & 630,113 & $1,399,486$ & $1,631,147$ & $3,214,928$ \\
\hline
\end{tabular}

\section{CONCLUSIONS}

The application of the extreme value theory to statistics allows us to fit models to data from the upper tail of a distribution. We have fit generalized extreme value and generalized Pareto data distribution to rice loss caused by typhoons in Taiwan by using the block-max and peak-over-threshold approaches to extreme value modeling. The appropriateness of the upper tail fitting to loss data is evaluated by comparing with standard parametric modeling based on lognormal and gamma distributions. The extreme value theory outperforms classical parametric fits. Furthermore, one of the main advantages of the Extreme Values model is that it gives a theoretical motivation for the procedures and satisfactory ways to find distributions for maxima, and to test the model. The results show that the loss distribution process is heavy-tailed, which implies that it is also non-normal, and the assumption of normality imposes a smaller threshold value even with the presence of fat-tails. From the risk management's point of view, separate margins should be imposed for these estimators, reflecting the risk that is inherent in the respective losses. These results could help the authorities and agriculture risk managers to check the applicable loss-compensation regulations. It is expected that, with favorable developments in policies on agricultural natural disaster aid projects, the authorities generally can be assured that operation of farmers in an agricultural economy will be in harmony with the stabilization program. It is also expected that the risk financing shocks caused by natural disasters will be reduced for 
both farmers and government, and should serve as guides for making further improvements. However, several problems remain along with this line for future research, one of which is the data dependent. We need to check the independency between frequency and severity of loss over time.

\section{REFERENCES}

Abbaspour, K. C., Hall, J. W., \& Moon, D. E. (1992). A yield model for use in determining crop insurance premiums. Agricultural and Forest Meteorology, 60(1), 33-51.

Adams, R. M., Hurd, B. H., Lenhart, S., \& Leary, N. (1998). Effects of global climate change on agriculture: an interpretative review. Climate Research, 11, 19-30.

Balkema, A. A., \& de Haan, L. (1974). Residual life time at great age. Annual Probability, 2, 792-804.

Chang, C. C. (2002). The potential impact of climate change on Taiwan's agriculture. Agricultural Economics, 27(1), 51-64.

Cheng, J. D., Huang, Y. C., Wu, H. L., Yeh, J. L., \& Chang, C. H. (2005). Hydrometeorological and landuse attributes of debris flows and debris floods during typhoon Toraji, July 29-30, 2001 in central Taiwan. Journal of Hydrology, 306(1-4), 161-173.

Gilli, M., \& Kellezi, E. (2006). An Application of Extreme Value Theory for Measuring Financial Risk. Computational Economics, 27, 207-228.

Hansen, L. M. (2004). Economic damage threshold model for pollen beetles (Meligethes aeneus F.) in spring oilseed rape (Brassica napus L.) crops. Crop Protection, 23(1), 43-46.

Jenkinson, A. F. (1955). The Frequency Distribution of the Annual Maximum (or minimum) Values of Meteorological elements. Quarterly Journal of the Royal Meteorology Society, 87, 145-158.

Ji, Q. Z., Hayakawa, S., Yamamoto, H., Okada, N., \& Tatano, H. (2002). Comparisons of agricultural damages by typhoon 9917, 9919 and 9918. Japanese Journal of Crop Science, 71(2), 239-249.

Jorion, R. (2007). Value at Risk. New York, NY: McGraw-Hill.

Lai, L. H. \& Wu, P. H. (2006). Using generalized Pareto distribution to estimate paddy rice loss from major agriculture disasters in Taiwan. Paper presented at International Conference on Business and Information (BAI) 2006, Singapore. 
Lai, L. H. \& Wu, P. H. (2007). An Extreme Value Analysis of Taiwan's Agriculture natural Disaster Loss Data. Paper presented at International Conference on Business and Information (BAI) 2007, Tokyo, Japan.

Lai, L. H. \& Wu, P. H. (2007). Risk management under extreme loss. Paper presented at WiCom 2007 International Conference on Wireless Communications, Networking and Mobile Computing, Shanghai, China.

Lai, L. H. \& Wu, P. H. (2008). Estimating the threshold value and loss distribution: Rice damaged by typhoons in Taiwan. African Journal of Agricultural Research, 3(12), 818-824.

Lansigan, F. P., De los Santos, W. L., \& Coladilla, J. O. (2000). Agronomic impacts of climate variability on rice production in the Philippines. Agriculture, Ecosystems \& Environment, 82 (1-3), 129-137.

Larsson, H. (2005). A crop loss model and economic thresholds for the grain aphid, Sitobion avenae (F.), in winter wheat in southern Sweden. Crop Protection, 24(5), 397-405.

Lin, M. L., \& Jeng F. S. (2000). Characteristics of hazards induced by extremely heavy rainfall in Central Taiwan-Typhoon Herb. Engineering Geology, 58(2), 191-207.

Luo, Y., Teng, P. S., Fabellar, N. G., \& TeBeest, D. O. (1998). Risk analysis of yield losses caused by rice leaf blast associated with temperature changes above and below for five Asian countries. Agriculture, Ecosystems \& Environment, 68(3), 197-205.

Muralidharan, K., \& Pasalu, I. C. (2006). Assessments of crop losses in rice ecosystems due to stem borer damage (Lepidoptera:Pyralidae). Crop Protection, 25(5), 409-417.

Oerke, E. C., \& Dehne, H. W. (2004). Safeguarding production-losses in major crops and the role of crop protection. Crop Protection, 23(4), 275-285.

Pickands, J. (1975). Statistical Inference Using Extreme Order Statistics. Annals of Statistics, 3, 119-131.

Reiss, R. D., \& Thomas, M. (1997). Statistical Analysis of Extreme values. Basel: Birkhäuser Verlag.

Rosenzweig, C., Tubiello, F. N., \& Goldberg, R. (2002). Increased crop damage in the US from excess precipitation under climate change. Global Environmental Change, 12(3), 197-202. 
Singleton, G. R., Sudarmaji, Jacob, J., \& Krebs, C. J. (2005). Integrated management to reduce rodent damage to lowland rice crops in Indonesia. Agriculture, Ecosystems \& Environment, 107(1), 75-82.

Taylor, B. (1996). Scirpophaga incertulas (Walker) (Lepidoptera: Pyralidae) and deepwater rice - an integrated view. Crop Protection, 15(7), 649-655.

von Mises, R. (1936). La distribution de la plus grade de n valeurs. Rev. Math. Union Interbalcanique, 1, 141-160.

Wu, C. C., \& Kuo, Y. H. (1999). Typhoons affecting Taiwan: Current understanding and future challenges. Bulletin of the American Meteorological Society, 80(1), 67-80.

Yamagata, Y., Wiegand, C., Akiyama, T., \& Shibayama, M. (1988). Water turbidity and perpendicular vegetation indices for paddy rice flood damage analyses. Remote sensing of enviorment, 26(3), 241-251.

Yamamoto, H., Iwaya, K., Suzuki, K., Hayakawa, S., \& Suzuki, Y. (2000). Agriculture disaster and salt damage in rice caused by typhoon 9918 in Kyushu and Yamaguchi districts. Japanese Journal of Crop Science, 69 (3), 424-430.

Yamamoto, H., \& Iwaya, K. (2006). Salt wind damage on rice by typhoon 0415 (MEGI) on the Sea of Japan coastal region of Tohoku and Hokuriku districts. Japanese Journal of Crop Science, 75(1), 73-81. 
